\title{
Constructing A New Japanese Development Design Model "NJ-DDM"
}

\author{
- Intellectual Evolution of an Automobile Product Design -
}

\author{
Kakuro Amasaka \\ Aoyama Gakuin University \\ Graduate School of Science and Engineering \\ Kanagawa-ken, Japan \\ kakuro_amasaka@ise.aoyama.ac.jp
}

\begin{abstract}
The author consructs a New Japanese Development Design Model “NJ-DDM" utilizing CAE to realize "simultaneous achievement of QCD" (quality, cost and delivery) in automobile development design by evolvinging the super-short-term development design process. Base on this, the author verifies the validity of this model in intellectual evolution of an automobile product design.
\end{abstract}

Keywords-New Japanese Development Design Model, Product design, CAE

\section{INTRODUCTION}

The study subject is the establishment of design quality assurance system employing numerical simulation (CAE) in intellectual evolution of an automobile product design. A new area of interest in the study of development design to win the "worldwide quality competition" is the shift of business process management from "experimental evaluation based on tests and prototypes" to "predictive evaluation based on highly reliable CAE analysis" [1,2].

Against this background, the author has recognized the necessity for making advancement in the development design, which is the core factor for strengthening product development ability, and thus constructs a new development design model, New Japanese Development Design Model "NJ-DDM" utilizing CAE. To be specific, NJ-DDM consists of Total QA (Quality Assurance) High Cyclization Business Process Model, Total Intelligence CAE Management Model, Highly Reliable CAE Analysis Technology Component Model, and Total Intelligence CAE Approarch Model [1-5].

Based on this, the author will evolves the conventional development design process to realize the simultaneous achievement of QCD required by evolving the super-shortterm development design process for global production strategy. The author later verifies the validity of NJ-DDM.

\section{DeVelopment Design of AutOMOBILES AND CAE}

\section{A. Current Issues in Japanese Automobile Industry}

At present, Japanese automobile manufactures are endeavoring to survive in the competitive market by expanding their global production and achieving "uniform quality worldwide and production at optimum locations”. In the midst of rapid change of management technologies, a key challenge facing the automobile manufacturers is to construct a new Japanese development design model which provides the latest, highly reliable, customer-oriented products ahead of their competitors so that they can survive the worldwide quality competition [1,2].

As for the pending issue of management technology in the development/production process, a cycle of "experiment -prototyping-evaluation” has been repeatedly carried out in order to prevent "scale-up effect" between the stages of experiment and mass production. As a result, the cost increased and development time prolonged. Therefore, a evolution in the conventional development design process is currently needed.

\section{B. CAE Application and Isues in Development Design}

The time between product design and production has been drastically shortened in recent years with the rapid spread of global production. Quality assurance, or QA, has become increasingly critical. This makes it essential that the development design process - a critical component of QAbe evolved to ensure quality [3]. Fig. 1 shows the typical development design process currently used by many companies. The figure shows that companies first create product development design instructions based on market research and planning [4].

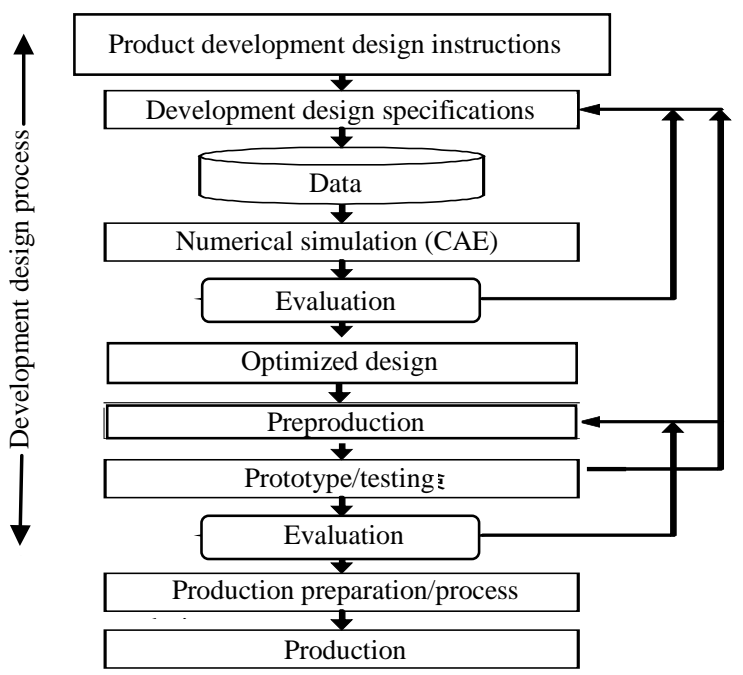

Figure. 1 CAE in the Development Design Process 
They then use these instructions to make development design specifications (drawings) and to promptly convert them to digital format so that they can be suitably processed and applied. The data is primarily used in numerical simulations known as computer-aided engineering, or CAE. $\mathrm{CAE}$ and other numerical simulations have been applied to a wide variety of business processes in recent years, including research and development, design, preproduction and testing/evaluations, production technology, production preparation, and manufacturing. These and other applications are expected to have effective results [3,6,7]. In this age of global quality competition, using CAE for predictive evaluation method in design work is expected to contribute a great deal to shortening development design time and improving quality [1-3].

However, in the case of automotive production, much of the development design process is guided by implicit experiential knowledge and rules of thumb, leading to prototype testing guided by repeated trial-and error efforts; in other words, a series of improvements based on conventional prototype testing methods. This not only prolongs the development design process, but also results in enormous testing costs. Previous forms of CAE analysis were not sufficiently precise, yielding figures that deviated as much as $10-30 \%$ from prototype testing evaluations (absolute values). This meant that CAE was hardly reliable enough to be an adequate substitute for prototype testing $[1,2]$. As a result, manufacturers were not able to cut out preproduction and prototype testing (a necessity for shortening development design time) despite the enormous amount of funds they invested in CAE development.

This means that many companies are now stuck with applying CAE only to the monitoring task of comparative evaluations of old and new products. The only way to get CAE analysis to function at a sufficient level and firmly establish it as part of (1) preventing recurrence of the pressing technical problem of bottlenecking and (2) the development design process for new products, is to make it more precise. Specifically, this means setting up highly reliable CAE analysis that reduce the deviation, or gap, with prototype testing evaluations (absolute values) to $5 \%$ or less $[2,3]$.

\section{CONSTRUCting A NeW JAPANESE DEVELOPMENT DESIGN MODEL}

In design and development for mass production, it is important to eradicate the repetitive trial-and-error testing of prototypes, and renovates low-productivity processes by introducing the latest CAE technology. In order to achieve this, the relevant departments must strategically cooperate to accumulate the necessary know-how [1,2]. Therefore, rather than adhering to the old systems, the author has constructed a New Japanese Development Design Model "NJ-DDM" utilizing CAE for the advanced development design shown in Fig. 2 as a way of overcoming these pressing problems in development design.

As the figure shows, technical issues that must be resolved by development design departments include development design process evolution, high accuracy of the prediction and control, computerization of business processes, and optimization of development design specifications. In terms of a methodology for resolving these issues, the author has created four core models: the Total QA (Quality Assurance) High Cyclization Business Process Model, the Total Intelligence CAE Management Model, the Highly Reliable CAE Analysis Technology Component Model, and the Total Intelligence CAE Approarch Model [1-5]. The organically integrated and intelligent application of these four models is essential. An overview of each is given below.

\section{A. Total QA High Cyclization Business Process Model}

As the first step, the author proposes the development design business process model. This model is created from the standpoint of verification/validation (divergence of CAE from theory and divergence of CAE from testing) in order to make possible highly reliable CAE analysis that is consistent with the market testing theory profile. The author

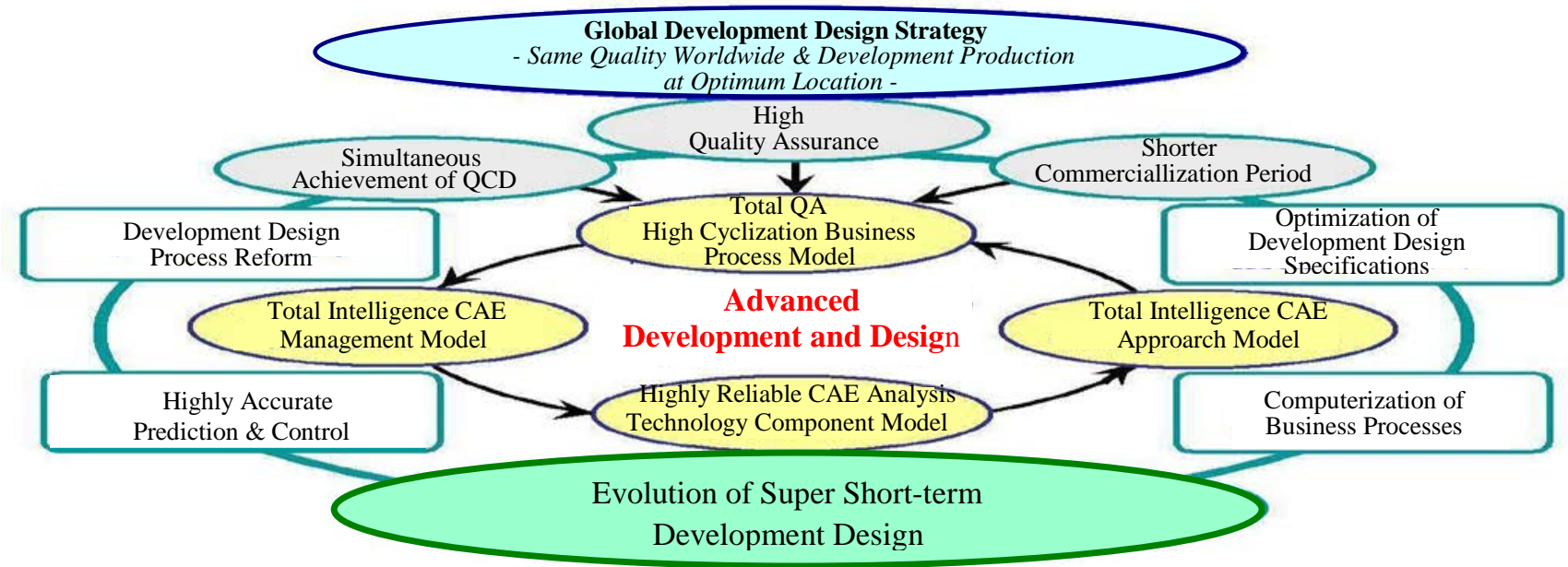

Figure. 2 New Japanese Development Design Model 


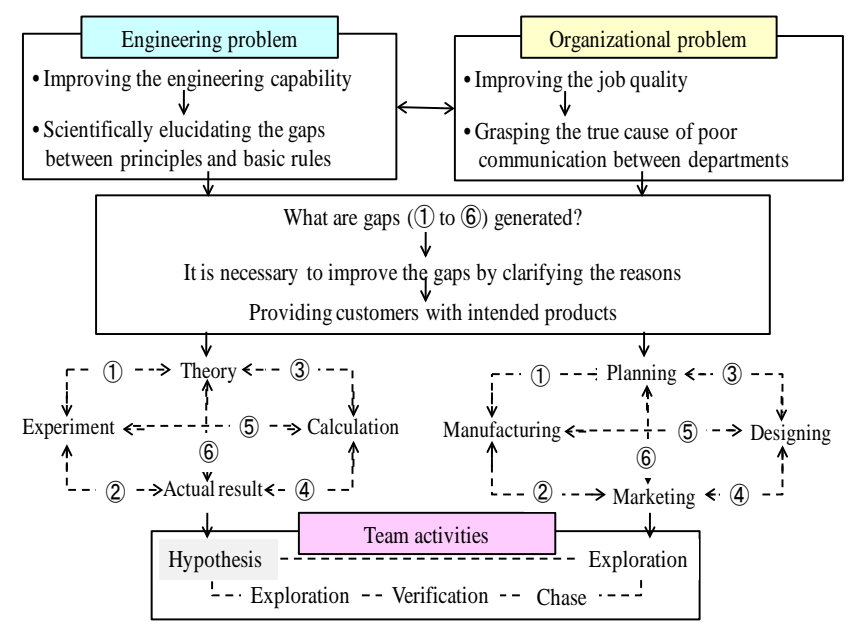

Figure. 3 Total QA High Cyclization Business Process Model

therefore recommend the introduction of the Total QA High Cyclization Business Process Model [2], which systematically realizes high quality assurance by incorporating analyses made via the core technologies of statistical science called Science SQC [8] as shown in Fig. 3.

For example, in order to solve the pending issue of a technology problem in the market, it is necessary to create a universal solution (general solution) by clarifying the existing six gaps (1) to (6)in Fig. 3) in the process consisting of theory (technological design model) experiment (prototype to production), calculation (simulation), and actual result (market) as shown on the lower left of Fig. 3. To accomplish this, the clarification of the six gaps (1) to (6) in the business processes across the divisions, shown in the lower right of Fig. 3 below, is of primary importance.

By taking these steps, the intelligent technical information owned by the related divisions inside and outside the corporation will be fully linked, thus evolving the business processes involved in development design.

\section{B. Total Intelligence CAE Management Model}

Following the above, the author proposes for Establishment of Total Intelligence CAE Management Model [2,3] shown in Fig. 4, which contributes to high quality assurance as well as QCD simultaneous achievement in automobile development design. As shown in this Fig. 4, many manufacturers are aware of the gap between evaluations of actual vehicles and CAE, and not fully confident in CAE results, they prefer to conduct Step (I) survey tests with actual vehicles rather than CAE evaluation. Even among leading corporations, Step (II) CAE utilization is limited to relative evaluation.

The author noticed a situation where, as shown in the figure, the application ratio of CAE to actual vehicles is $25 \%$ for surveys and about $50 \%$ for relative evaluation revealing the dilemma that the effectiveness of CAE invested for reduction in development time has not been fully utilized. Based on the above, in Step (III), as seen in

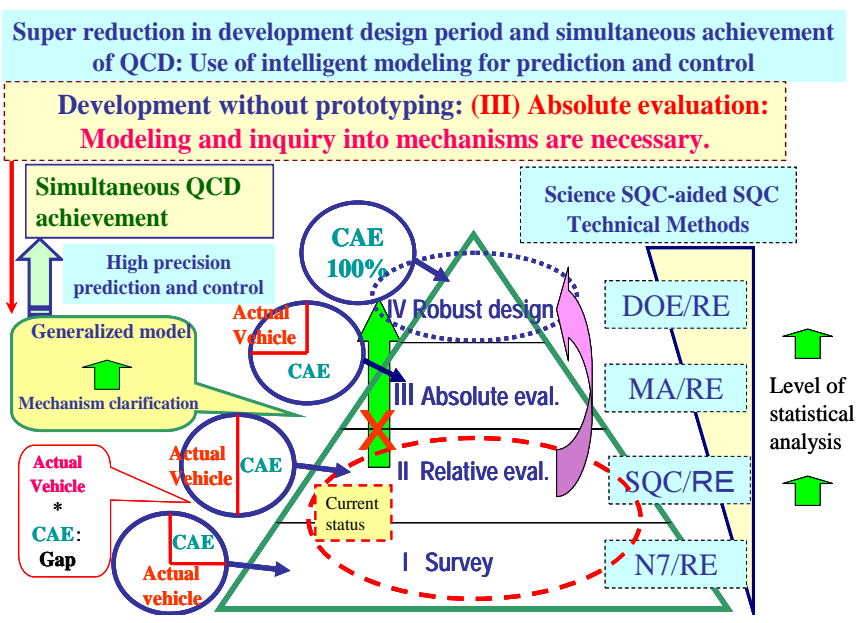

Figure. 4 Total Intelligence CAE Management Model

the figure, the mechanism of the pending technical problem was clarified through visualization technology, and the technical knowledge which enables absolute evaluation through the creation of generalized models was incorporated in the CAE software. As a result, it was confirmed that the accuracy of CAE analysis had improved and the application ratio of CAE had increased to $75 \%$.

Based on the technical analysis derived from Steps (I) to (III), Step (IV) further incorporated a robust design which takes into consideration the influential factors and contributing ratio needed for optimal design, thus enhancing the accuracy of CAE calculation, and demonstrating a remarkable increase in the ratio of CAE application.

\section{Highly Reliable CAE Analysis Technology Component Model}

The Highly Reliable CAE Analysis Technology Component Model shown in Fig. 5 was designed to make the shift from conventional prototype testing methods to effectively applying CAE in predictive evaluation methods. The comprehensive issuance of this model is essential to achieving the desired shift [1-3].

More specifically, the critical aspects of this model include (i) defining the problem (physically checking the actual item) in order to clarify the mechanism of the defect, using visualization technology to identify the dynamic

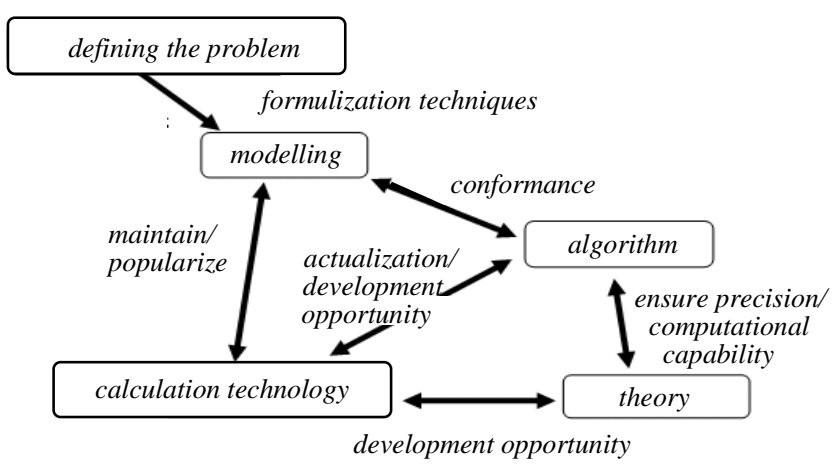

Figure. 5 Highly Reliable CAE Analysis Technology Component Model 
behavior of the technical issue; (ii) full use of formulization techniques to generate logical modelling (statistical calculations); (iii) constructing compatible algorithms; (iv) developing theories that ensure the precision of numerical calculations and sufficient computational capability; and (v) comprehensively putting the above processes in action using computer (selection of calculation technology).

\section{Total Intelligence CAE Approarch Model.}

Against the above background, as a fundamental solution to the automotive development and designing problem, the author created "Intelligence CAE Management System Approach Model" [2,3,10], as indicated in Fig. 6. As seen in the figure, first, the (A) actual vehicle and experiment (meaning bench evaluation tests using actual vehicles and parts) visualizes the dynamic behavior (tricky mechanism) of the problems. Next, by means of the (B) factorial analysis in which the unique technology and empirical technology are combined together with N7 (new 7 tools), RE (reliability), MA (multi variate analysis), and DE (design of experiment), the latent factors which induce oil leakage are investigated using actual vehicles and experiment procedures in an effort to clarify the mechanism.

Based on the knowledge obtained through the above steps, as well as the navigation process using Computer Graphics (CG) created by a combination of (C) experiments and $\mathrm{CAE}$, qualitative modeling of problems was conducted. Furthermore, for the purpose of accurately reproducing the mechanism, which has been grasped by an inductive approach of visualization experiments, quantitative modeling is conducted by means of (D) numeric value simulation. In the final stage, the (E) differential (gap) between the evaluation results of actual vehicles and experiment procedures (absolute value) and those of the CAE analysis (simulation value) was confirmed.

The target of the differential ratio of analysis precision is collective partnering is indispensable among the chief engineers (@), collaborating engineers ( $($ ), and assistants

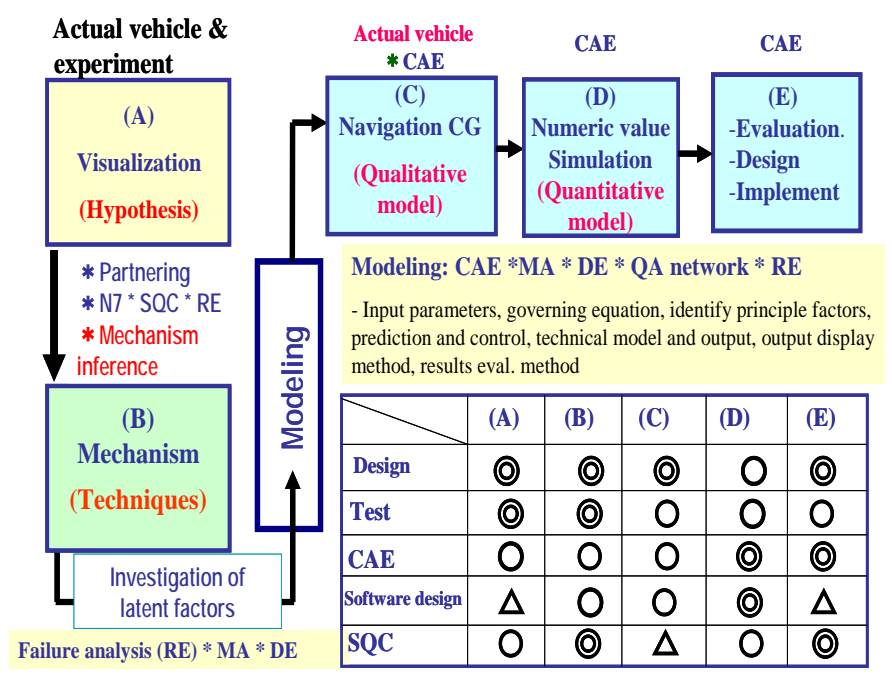

Figure. 6 Total Intelligence CAE System Approach Model between $1 \%$ and $2 \%$. To achieve such a target, an all-out, $(\triangle)$ who are involved in the design, testing, CAE analysis, CAE software development, and SQC throughout the process stages from (A) to (E) indicated in the figure.

Thus, this study uses visualization technology at the absolute evaluation stage to clarify the mechanisms involved in current technical problems and proposes a model for a highly reliable CAE analysis approach to enable absolute evaluation through the creation of generalized models.

\section{APPLICATION EXAMPLES}

The author was able to apply the NJ-DDM to critical development design technologies for automotive production, including predicting and controlling the special characteristics of automobile urethane seat foam molding [1], transaxle oil seal leakage [4,5,9], and loosening bolts [10]. In each of these cases as well, discrepancy was 3-5\% versus prototype testing.

\section{CONCLUSION}

In this paper, the author constructed the NJ-DDM as the research aimed at the intellectual evolution of development design processes to realize "simultaneous achievement of QCD" in these processes within the automotive industry. The validity of NJ-DDM has been verified with application examples.

\section{REFERENCES}

[1] K. Amasaka, Highly Reliable CAE Model, The Key to Strategic Development of Advanced TDS, Journal of Advanced Manufacturing Systems, vol.6, issue.2, pp.159-176, 2007.

[2] K. Amasaka, An Integrated Intelligence Development Design CAE Model Utilizing New JIT, Application to Automotive High Reliability Assurance, Journal of Advanced Manufacturing Systems, vol.7, no.2, pp.221-241, 2008.

[3] K. Amasaka, Proposal and Effectiveness of a High Quality Assurance CAE Analysis Model: Innovation of Design and Development in Automotive Industry, Current Development in Theory and Applications of Computer Science, Engineering and Technology, vol.2, no.1/2, August/November Issue, pp. 23-48, 2010.

[4] K. Amasaka, Constructing Optimal Design Approach Model: Application on the Advanced TDS, Journal of Communication and Computer, vol.9, no.7, pp. 774-786, 2012.

[5] K. Amasaka, T. Ito and Y. Nozawa, A New Development Design CAE Employment Model, The Journal of Japanese Operations Management and Strategy, vol.3, no.1, pp. 18-37, 2012.

[6] Y, Magoshi, H, Fujisawa and T, Sugiura, Simulation Technology Applied to Vehicle Development, Journal of Society of Automotive Engineers of Japan, vol. 53, no. 3, pp. 95-100, 2003.

[7] J. D. V. Leo, N, Annos and J, Oscarsson, Simulation Based Decision Support for Manufacturing System Life Cycle Management, Journal of Advanced Manufacturing Systems, vol. 3, no. 2, pp. 115-128, 2004.

[8] K. Amasaka, Science SQC, New Quality Control Principle: The Quality Strategy of Toyota, Springer, 2004.

[9] R. Akaiwa and K. Amasaka, CAE Analysis for Oil Leakage Mechanism of Transaxle Oil Seal, IOSR Journal of Computer Engineering, vol. 16, issue 4, pp.60-63, 2014.

[10] K. Hashimoto, T. Onodera and K. Amasaka, Developing a Highly Reliable CAE Analysis Model of the Mechanisms that Cause Bolt Loosening in Automobile, American Journal of Engineering Research, vol. 3, issue 10, pp. 178-187, 2014. 